\title{
Application of an Artificial Neural Network for Early and Accurate Diagnosis of Parkinson's disease
}

\author{
${ }^{1}$ Akshar Agarwal \\ ${ }^{1}$ Hamden High School, Connecticut, United States; \\ aakshar2000@gmail.com
}

\begin{abstract}
Parkinson's Disease (PD) is one of the most common neurological disorders, affecting more than ten million people globally. The hallmark symptoms of PD are tremors, limb rigidity, and imbalance. PD shares many of these symptoms with other disorders, making it difficult to diagnose. Furthermore, due to the lack of definitive laboratory tests, PD is poorly diagnosed with subjective examinations such as family history evaluations, resulting in high misdiagnosis rates. Recent research shows that an additional symptom, dysphonia, is uniquely present in over $80 \%$ of PD patients. Dysphonia is a speaking disorder caused by involuntary muscle movement and other neurological factors in PD. In this project, that unique symptom. A cross-validated neural network was programmed to deliver rapid and accurate diagnoses using biomedical voice data from 195 patients of varying statuses. This automated, machine-learning based PD diagnostic tool was successfully created and functions with over $95 \%$ accuracy. This rate includes nearly zero false negatives and few false positives, showing significant improvement over previous attempts which had misdiagnosis rates of nearly $20 \%$. A low probability of false negatives is favorable. The neural network was designed such that overfitting is avoided, and more features/data would further improve the algorithm's accuracy. An early and accurate diagnosis is critical for treating PD patients, and this project proposes a way to achieve that.
\end{abstract}

Keywords: Machine learning, Artificial intelligence, Parkinson's Disease, neural network, dysphonia

\section{Introduction}

Parkinson's Disease (PD) is one of the most common neurodegenerative disorders, affecting nearly $3 \%$ of adults aged 65 and older [2]. It is a common, yet highly complex disease which results from both genetic and environmental factors. PD has severe symptoms, most notably tremors, limb rigidity, and gait issues $[2,3]$. The disease is primarily characterized by the loss of dopaminergic neurons the midbrain [2]. Despite its prevalence, there currently exists no objective method of determining whether a patient is affected by PD. The diagnosis rests upon subjective evaluations such as family history evaluations, handwriting examinations, and response to medication. Although new imaging techniques have been developed to aid in the diagnosis of PD, there are still no proper diagnostic tools. Furthermore, many other diseases present with similar symptoms (MS, Fibromyalgia, Lewy-Body dementia), causing misdiagnosis rates to remain significantly high at approximately $24 \%$ [3]. Recent studies, including one performed by Kish et al, have found that drastic neuronal depletion has been observed by the time that motor symptoms emerge. Because dopaminergic loss occurs rapidly when left untreated, an early and accurate diagnosis of PD is essential [4]. An early and accurate diagnosis is essential to patient outlook, in terms of both health and 
finances. Medication can aid in slowing neurological damage or controlling movement, and economically speaking, treatment costs grow exponentially with the severity of the disease. Recent research suggests that $80-95 \%$ of PD patients present with dysphonia, a speaking disorder, in the earliest stages [1]. Although the exact mechanism remains unknown, dysphonia is the result of various neurological and physical influences which occur with Parkinson's Disease. Because vocal impairment is one of the earliest indicators of PD, and voice samples can be collected non-invasively, utilizing voice-based diagnostic tools would provide a significant improvement over current diagnostic techniques. In this project, an artificial neural network was created to diagnose PD by utilizing voice samples of healthy and diseased patients.

\section{Algorithm Development}

The Statistics and Machine Learning Toolbox was used within MATLAB to develop an Artificial Neural Network which can classify patient condition given voice data.

\subsection{Data}

The dataset used here consists of 195 sustained vowel phonations from 31 patients, 23 of whom have been diagnosed (in various stages) with Parkinson's Disease. The patients' ages ranged from 46 to 85 years, as shown in Figure 2.1, and the time since diagnosis ranged from 0 to 28 years, as shown in Figure 1. The data was collected and provided by request from Dr. Max Little, in collaboration with the National Center for Voice and Speech (Denver, CO). The speech signals were recorded primarily using the Kay Pentax MultiDimensional Voice Program [1].

Age Distribution of All Subjects

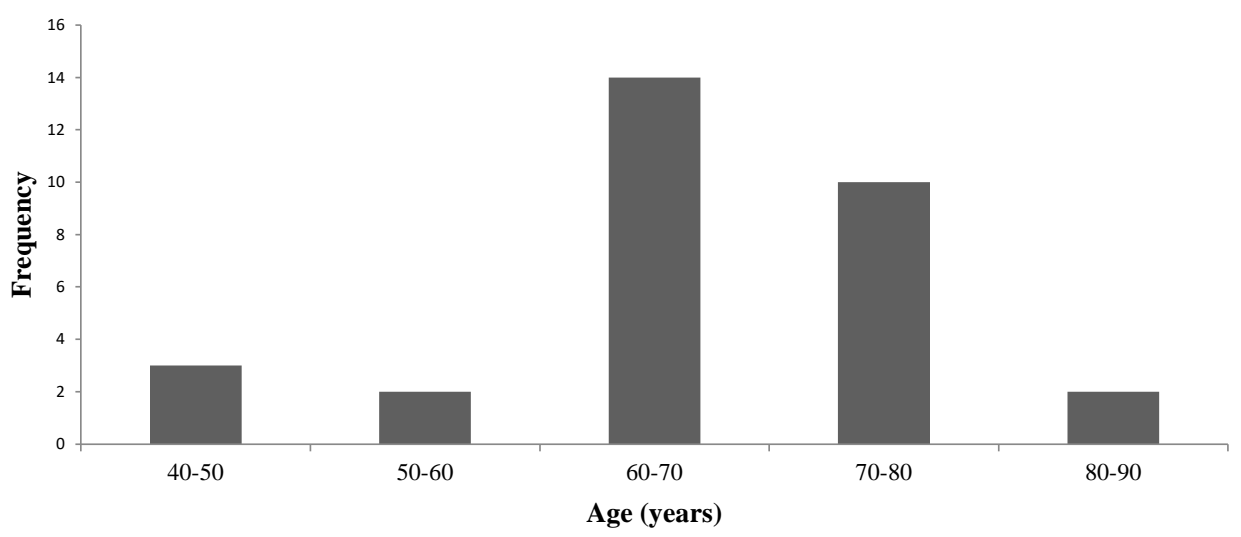

Figure 1: A histogram showing the distribution of subjects' ages.

\subsubsection{Features}

The data consists of 22 features, including various measures of fundamental frequency, amplitude, et cetera. Other important features included jitter (relative changes in fundamental frequency), shimmer (relative changes in amplitude across vocal cycles), and several values which quantify the noise-toharmonics ratios.

\subsection{Neural Network}

An artificial neural network (ANN) was designed here to classify subject condition as 1 (diseased) or 0 (healthy). A multilayer perceptron was created and validated with leave-one-out (LOO) cross-validation 
and dropout layers. The LOO cross-validation entails training on $\mathrm{n}-1$ trials and then testing on the remaining 1 trial for $\mathrm{n}$ iterations. Dropout entails selectively dropping or ignoring random neurons and their inputs. This is done to reduce both variance and bias in efforts of creating an optimal classifier which neither overfits nor underfits $[6,7]$. Other basic architectural properties include a logistic sigmoidal transfer function between the hidden layer and output layer to force a binary output.

\subsection{Bayesian Optimization}

ANNs are commonly referred to as black box functions. There are many parameters whose influence on overall error is largely unpredictable. While it is not necessarily true that all ANNs operate as such, the model created here is certainly not convex. Its global minimum is difficult to locate, and thus Bayesian optimization, as described by Snoek et al. [5], was utilized for hyperparameter selection and model evaluation. Grid searches are computationally intensive and grow exponentially with more parameters. On the other hand, this method provides a more logical search and can be run with less resources. Bayesian optimization involves fitting a Gaussian process to the model to predict which parameters will return more optimal accuracies/errors. Figure 2 shows the resulting model of the objective function which was obtained by tracing outputs with two variable hyperparameters. This process calculates the expected improvement across parameters and seeks to minimize the value of the objective function (error).

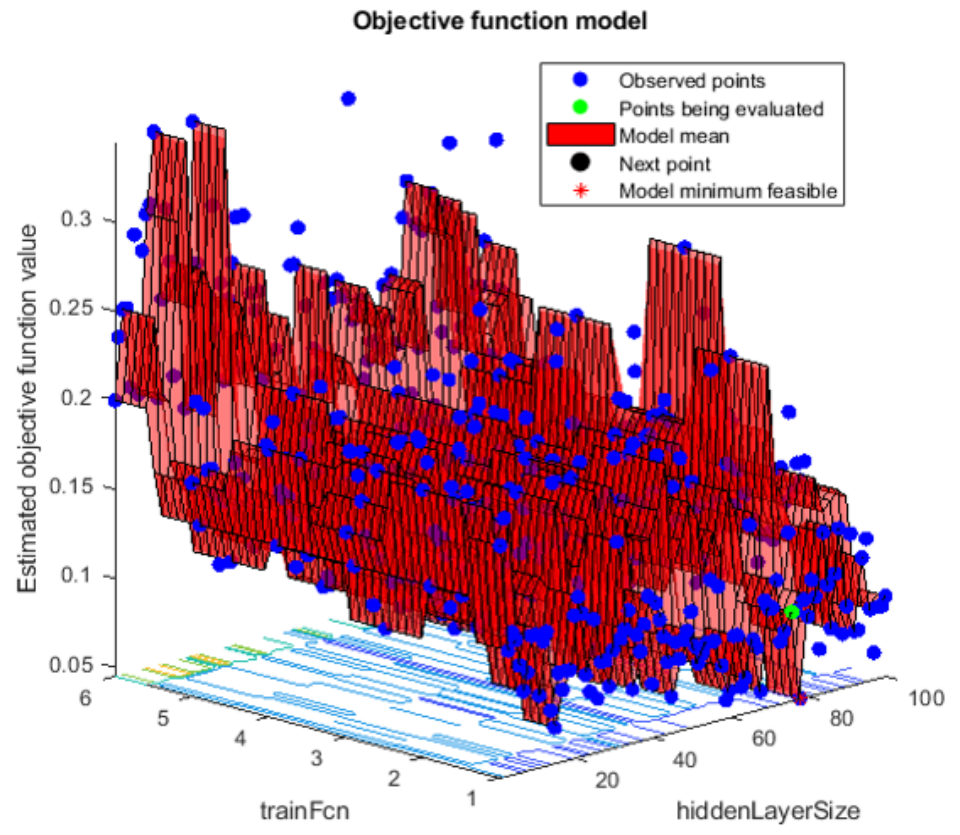

Figure 2: A model of the Artificial Neural Network obtained through the completion of one thousand iterations of Bayesian Optimization.

\section{ANN Performance and Results}

The optimal ANN architecture was determined to consist of a single hidden layer with 78 neurons. The transfer function between the input and hidden layers was the Elliot symmetric sigmoidal function, and the Levenberg-Marquardt algorithm was utilized for backpropagation.

$$
x_{k+1}=x_{k}-\left[J^{t} J+\mu I\right]^{-1} J^{t} e
$$


The Levenberg-Marquardt algorithm is one of the fastest and most accurate methods of backpropagation [5]. As seen in the equation above, $J^{t} J$ represents the Hessian matrix, and $J^{t} e$ is the gradient where $J$ is the Jacobian matrix of first derivatives. The Hessian and Jacobian matrices describe the local curvature of a multivariate function. $\mu$ is a scalar value which helps model the algorithm after Newton's method, which is particularly fast while approaching a global error minimum. This algorithm transforms from gradient descent to a quasi-Newton BFGS algorithm as $\mu$ decreases.

With that architecture, the cross-validated artificial neural network yielded an overall accuracy of 95.9\%, as seen in the confusion matrix in Figure 3.

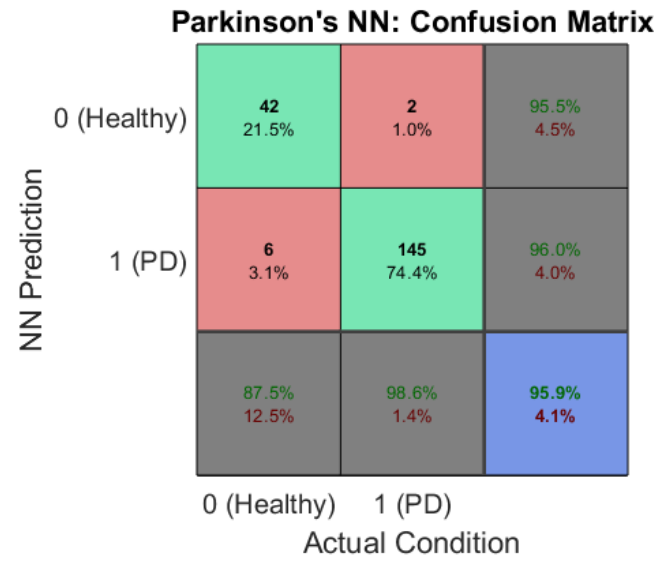

Figure 3: A confusion matrix displaying the accuracy of the ANN by class.

A high probability of detection is the obvious objective, but a low false-negative rate is especially favorable in medical settings. The model created here functions with a false-negative rate of just $1.4 \%$, indicating that patients with Parkinson's disease are not given an inaccurate diagnosis (healthy) at a statistically significant rate.

\section{Conclusion}

Parkinson's Disease is one of the most prevalent neurodegenerative disorders, and yet its complexity often leads to misdiagnosis and defers treatment for years. Detecting Parkinson's Disease in its early stages is crucial, and the nature of the disease does not allow for physicians to detect it efficiently. Misdiagnosis rates are significantly high, and this creates issues for patients both economically and

\section{REFERENCES}

[1] Little, M. A., McSharry, P. E., et al. (2009). Suitability of dysphonia measurements for telemonitoring of Parkinson's disease. IEEE Transactions on Bio-Medical Engineering, 56(4), 1015.

[2] Sewall G. K., Jiang J., Ford C. N. Clinical evaluation of Parkinson's-related dysphonia. Laryngoscope. 2006;116(10):1740-1744.

[3] Hughes AJ, Daniel SE, Kilford L, Lees AJ. Accuracy of clinical diagnosis of idiopathic Parkinson's disease: a clinico-pathological study of 100 cases. J Neurol Neurosurg Psychiatry. 1992;55(3):181-184. 
Akshar Agarwal; Application of an Artificial Neural Network for Early and Accurate Diagnosis of Parkinson's disease. Transactions on Machine Learning and Artificial Intelligence, Volume 6 No 3 June (2018); pp: 20-24

[4] Kish SJ, Shannak K, Hornykiewicz O. Uneven pattern of dopamine loss in the striatum of patients with idiopathic Parkinson's disease: pathophysiologic and clinical implications. N Engl J Med. 1988;318(14):876-880.

[5] MathWorks, (2017). Statistics and Machine Learning Toolbox: User's Guide (R2017b).

[6] Srivastava, Nitish, et al. "Dropout: A simple way to prevent neural networks from overfitting." The Journal of Machine Learning Research 15.1 (2014): 1929-1958.

[7] Kohavi, Ron. "A study of cross-validation and bootstrap for accuracy estimation and model selection." ljcai. Vol. 14. No. 2. 1995. 